\title{
Exercise-induced changes of CSF vascular endothelial growth factor in adult chronic hydrocephalus patients
}

\author{
Mark Gregory Luciano ${ }^{1 *}$, Jun Yang ${ }^{1}$, Kaitlyn J Shanahan ${ }^{1}$, Leah P Shriver ${ }^{2}$ \\ From Hydrocephalus 2015 \\ Banff, Canada. 18-21 September 2015
}

\section{Background}

Vascular endothelial growth factor (VEGF) is a growth factor demonstrated to play a key role in cerebral angiogenesis and neurogenesis. It has been considered a critical component in hippocampus neurogenesis and memory formation and has been observed to increase in the rat hippocampus after exercise. In a previous study, we found increases in VEGF receptor and/or ligand in an experimental model of chronic e hydrocephalus in several brain areas and cerebrospinal fluid (CSF), suggesting a role in the adaption to chronic hypoxia. Here we investigate the ability of moderate exercise to increase CSF-VEGF levels in adult chronic hydrocephalus patients.

\section{Methods}

Lumbar CSF samples were collected from 17 normal pressure hydrocephalus (NPH) patients over 5 hours in 1-h intervals. During CSF collection, 11 patients (exercise group) underwent a standard in-room physical therapy session; 6 patients (no-exercise group) did not undergo a physical therapy session. CSF-VEGF levels were evaluated for increase related to exercise and the clinical response to CSF drainage.

\section{Results}

CSF-VEGF levels in the exercise group demonstrated significant increases 1 -3hrs post-exercise compared with the levels 1 - $2 \mathrm{hrs}$ pre-exercise $(\mathrm{p}=0.04)$, and also showed significantly higher levels than the no-exercise groups $(\mathrm{p}=0.03)$. While patients who clinically improved with CSF removal did not demonstrate an increase in CSFVEGF levels, those who did not clinically improve had higher CSF-VEGF levels after exercise. The post-exercise CSF-VEGF level in the group that did not clinically improvement was significantly higher than both their own pre-exercise level $(\mathrm{p}=0.02)$ and also higher than that seen in the clinically improving group $(\mathrm{p}=0.05)$ after exercise.

\section{Conclusions}

CSF-VEGF levels can increase after moderate exercise even in elderly hydrocephalus patients. This suggests a potential benefit of exercise in benefiting some of these patients may exist via a central VEGF mechanism. Increased VEGF levels after exercise in patients who showed no improvement with CSF drainage suggest that vascular injury may play a role in this group's pathophysiology.

\section{Authors' details \\ ${ }^{1}$ Cleveland Clinic, USA. 'University of Akron, Depts. Chemistry and Biology, USA.}

Published: 18 September 2015

doi:10.1186/2045-8118-12-S1-058

Cite this article as: Luciano et al.: Exercise-induced changes of CSF vascular endothelial growth factor in adult chronic hydrocephalus patients. Fluids and Barriers of the CNS 2015 12(Suppl 1):O58.

* Correspondence: mark.luciano.md.phd@gmail.com

${ }^{1}$ Cleveland Clinic, USA

Full list of author information is available at the end of the article 